\title{
Using genetics to guide treatment of arterial hypertension with Rostafuroxin
}

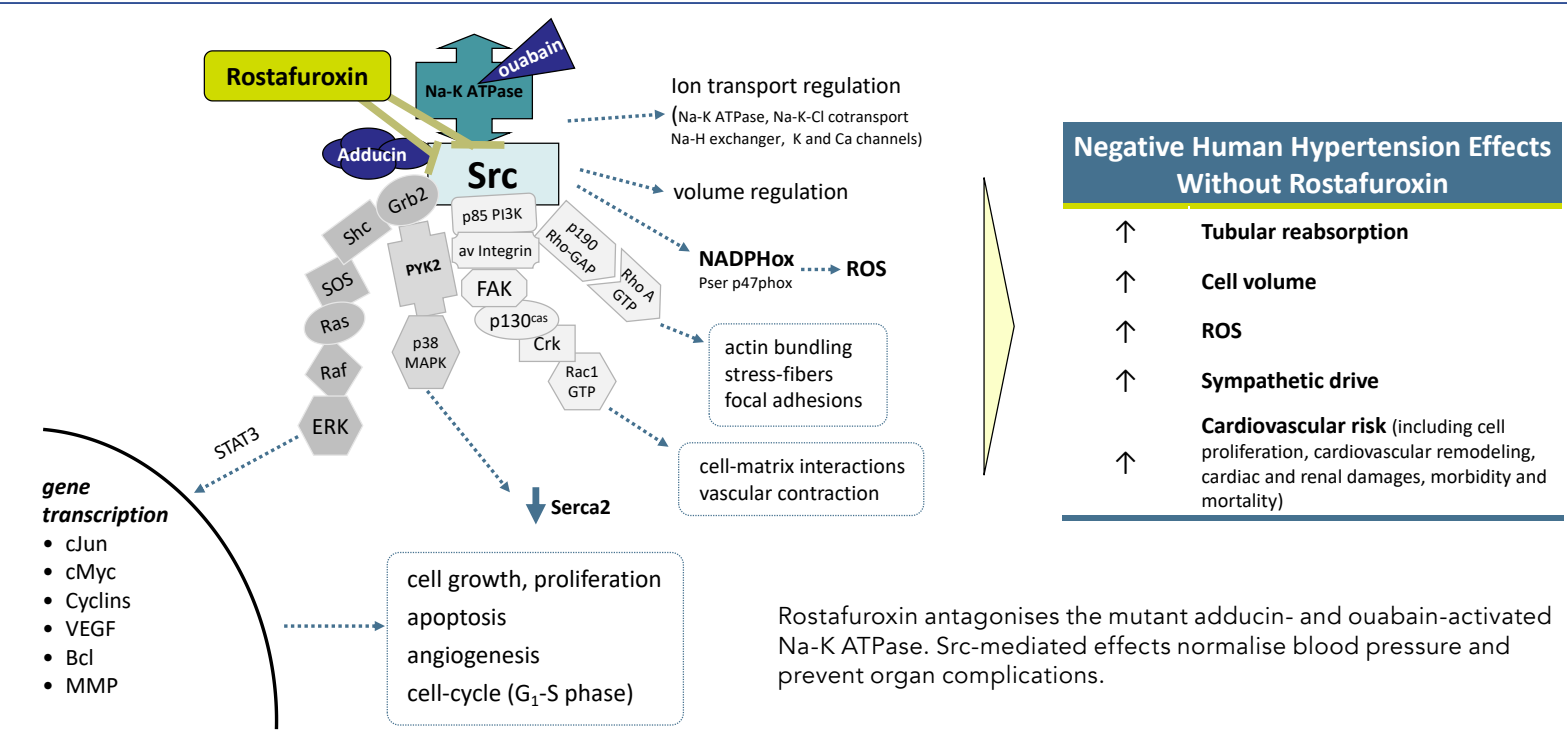
Primary hypertension is a
major health concern and can cause serious cardiac, respons brain complications worldwide health burden and costs. The development of hypertension is triggered by genetic factors that, in turn, are modulated by biological and environmental factors. Many drugs are available for the treatment of hypertension, but none target hy underlying genetic mechanisms. During the in treatment efficacy has been achieved. Dr Giuseppe Bianchi, Professor Emeritus at the Vita-Salute San Raffaele University, Milan, and CSO at Windtree Therapeutics, aims to explore the relationship between an individual's genetic rostafuroxin that 'selectively' targets them.

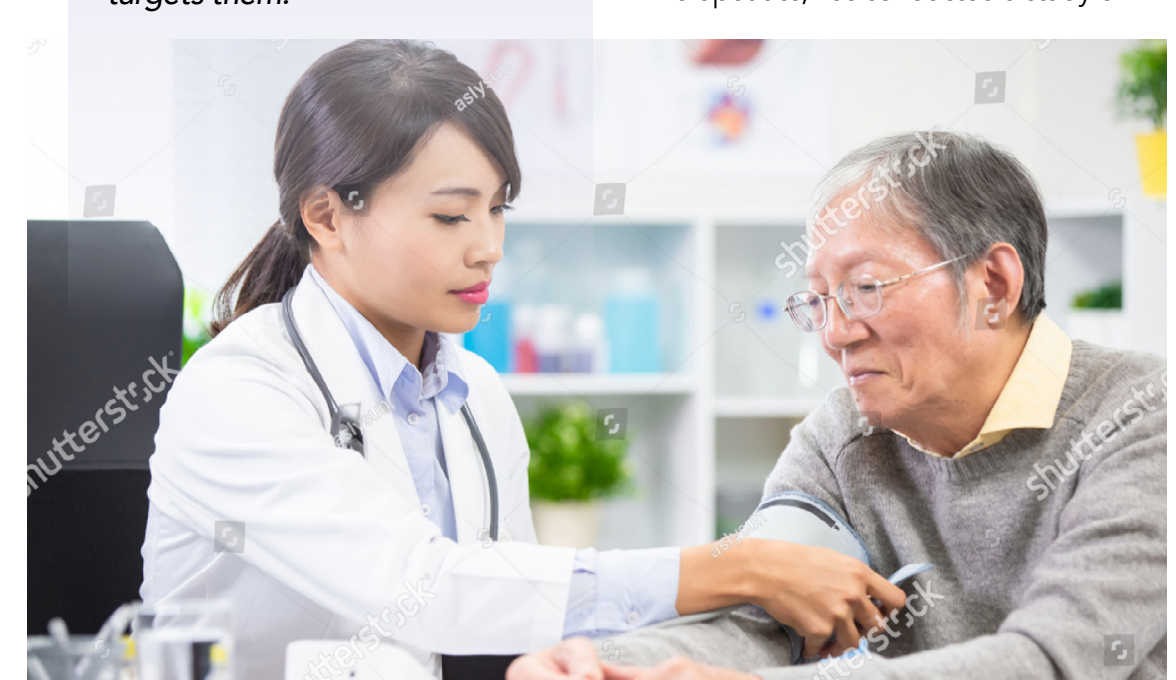

rats infused with ouabain, and his findings to fully protect the abiliy of current drugs complications, even when they are able to normalise blood pressure. Ouabain is a steroid hormone which is able to improve the contraction of the herrt muscle and increase blood pressure, making it a promising target for treating hypertension.

\section{PRECISION MEDICINE}

Drugs interact with other body molecules to exert their wanted or unwanted effects. These molecules are encoded by someone's genetic makeup and may vary from person to person (gene variants). Some of these gene variants may trigger the the presence of specific biological and

Considering the well-known heterogeneity of both the genetic mechanisms of hypertension and the avallable drug's blood pressure response, genetic mechanism(s) at work in a specific subset of patients, and 2) the therapeutic efficacy of any medication that is able to selectively inhibit these mechanisms in this subset of patients.

Over the last 30 years, many genetic variants $(>1,000)$ have been found to be associated with blood pressure level. Unfortunately, their causality in triggering is still open to discussio. The damage is sin open to dsccussion. The che, Dr is stil operto dissussion. Therefore, Dr associated genetic characteristics, with each group being assigned to with a characteristics. The selective therape tic efficacy of medication in this subset of patients is useful to demonstrate both its ability to cure these patients and the disease causality of the gene variants at work in them.

Precision medicine is based on the principle that curative therapy has to selectively target the molecular 'abnormality(ies)' causing the disease, while minimising the interaction with the other body molecules. The molecular abnomalties can be caused by different gene varants in different people, hence

\section{THE ADVANTAGES OF} OSTAFUROXIN

Dr Bianchi explains that one particular mportant in taking the first steps tow Unlike current drugs used to treat hypertension, rostafuroxin is able to ddress the genetic causal mechanisms underlying the condition in a subset of patients, and this has been demonstrated n animal and human trials. Genetic testing must be used to identify the patients who are most likely to respond to treatment.

The drug works by selectively inhibiting common step of the pathways leading triggered by endogenous ouabain (EO) and mutant adducin. It disrupts the binding to the CSrc-SH2 domain of mutan a-adduch and $10^{-11} \mathrm{M}$, being an wild addun or no being inefective pump or on the other 34 receptors or proteins involved in cardiovascular regulation up to $10^{-5}$. In other words, it inhibits the effects of EO, a type of Rostafuroxin has been developed with a is series of studies across different settings complications have significant animals and humans, together health and economic costs.

people with hypertension Levels of $\mathrm{EO}$ in the body tissues are controlled by certain variants of the genes (LSS, HSD3B1 and MDRT) wh thafect boh, Hs synthesis and transpor. Me averiostas gene, which are associated with body sodium and blood pressure changes bo in rats and in patients.

When $E O$ is given to rats, they develop ienal dood pressure and cardiac and are prevented by administration of ostafuroxin, while amlodipine (a first-line drug treatment for hypertension) only normalises blood pressure. or absence of the above 


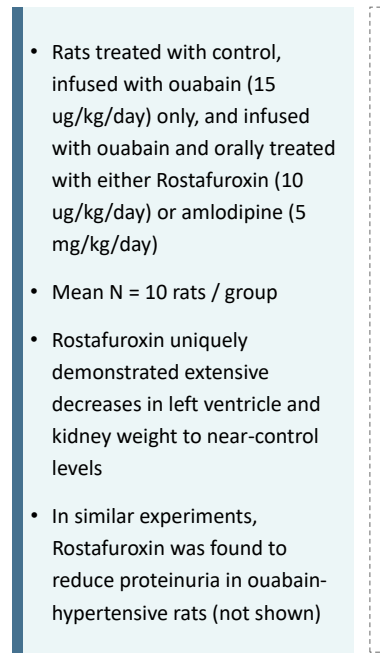

have been done, involving 84 healthy volunteers and 802 hypertensive patients. CLINICAL TRIALS

One Phase II trial was the OASIS-HT trial. This was a double-blind, crossover study, meaning that patients are given either the test dng or the control drig for the first to the other grou for the remainder of the study Over 400 Caucasian people with hypertension were involved in the trial, and the results showed that rostafuroxin did reduce blood pressure only in a subset of naive patients carrying the above gene variants either alone or grouped in what the researchers established as genetic profile 2. Profile 2 consists of variants of $\alpha$-adducin (ADD1) rs4961), $y$-adducin (ADD3 rs3731566), lanosterol synthase (LSS rs2254524), 3 Bhydroxysteroid dehydrogenase/ $\delta(5)-\delta(4)$ isomerase type 1 (HSD $3 \mathrm{~B} 1$ rs 10923835). and ATP-binding cassette subfamily $B$ member 1 (ABCB1MDR1 rs 1045642) genes, the last three being involved
regulation of EO tissue levels.

The PEARL-HT trial (the second proofff-concept phase llb trial involped 279 patients (172 Caucasians and 107 Chinese) with untreated hypertension who fitted the previously identified genetic profile 2 and carried the LSS gene mutation for $\mathrm{EO}$. Compared to the control group receiving losartan, another commonly used antihypertensive drug the Caucasian group given $0.05 \mathrm{mg}$ rostafuroxin showed a very remarkable blood-pressure fall (23-27 mmHg), starting from baseline levels around $150 \mathrm{mmHg}$. In contrast, the bloodpressure response to losartan was significantly lower

Conversely, the blood-pressure-lowering effect of rostafuroxin was much less in

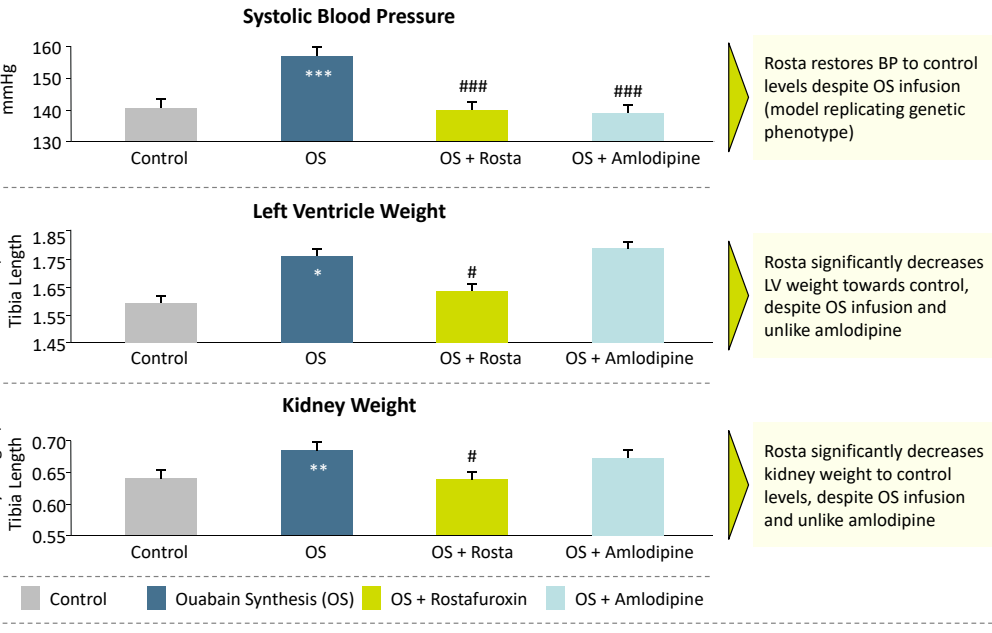

ction in antihypertensive rats.

the Chinese groups. It is well known that different ethnicities metabolise drugs at different rates. A number of arguments support the notion that in the presence of such low doses (0.05-0.5mg daily). rostafuroxin may be metabolised by time to pat This hypothesis is going to tested in future studies.

\section{FUTURE DIRECTIONS}

Primary hypertension and its associated economic costs. As mentioned above, the current drug treatments have many limitations that could be overcome by using a more targeted approach

safely reduce blood pressure, as well as protect organs from damage caused by hypertension, somehing that accounts for around $10 \%$ of overall global health expenditure.

While the selective effect of rostafuroxin on organ damage and blood pressure has has not been tested in human subiects. However, there are many indications that heart or kidney damage caused by hypertension is associated with the gene variants included in the profile 2 subset of individuals. Therefore, it is likely that these protective effects of rostafuroxin will be translated to patients.

\section{Unlike current drugs used to treat} hypertension, rostafuroxin is able to address the causal mechanisms underlying

\section{the condition in a subset of patients.}

that may offer a more effective an resource-efficient approach Dr Bianchi emphasises that rostafuroxin is a more efficient treatment option on blood pressure and organ damage for about $25 \%$ of the hypertensive population who have mutations in the adducin gene and in genes that regulate $\mathrm{EO}$ synthesis and transport. The drug is able to

Many patients currently receive a the desired response Rostafuroxin has the potential to be a ingle-drug replacement for these combination therapies in people with specific gene variants, particularly for those with resistant hypertension, meaning better outcomes for patients and more cost-effective use of medications.

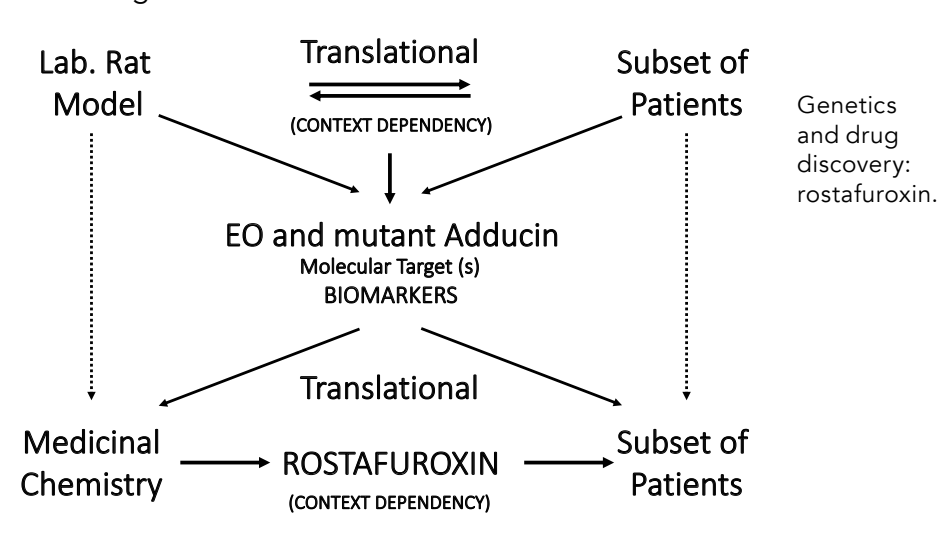

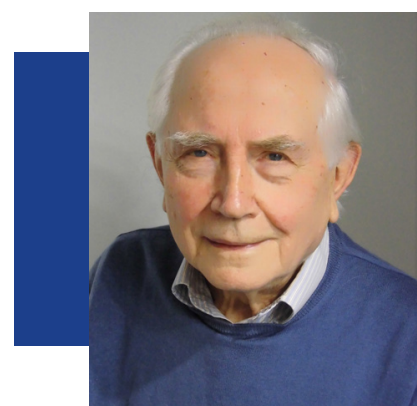

\section{Behind the Research}

\section{Dr Giuseppe Bianchi}

E: bianchi.giuseppe@hsr.t T: $\quad+393488545560 \quad$ W: orcid.org/0000-0002-8365-3618

\section{Research Objectives}

Dr Bianchi studies antihypertensive agent rostafuroxin.

\section{Detail}

Division of Nephrology and Hypertension

San Raffaele Hospital, via Olgettina, Milano, Italy

Bio

Guseppe Bianchi trained in cardiovascular-renal pathophysiology and biochemistry at the Universities of Milano, Parma, Padua, London, and Glasgow, with a focus on cardiology and nephrology. He is Hon MD at the Universities of Berlin and Uppsala, and won awards from British, American, and Italian societies of hypertension, the USA National Kidney Foundation and other scientific Raffaele University, and CSO at Windtree Therapeutics.

Over the years, Dr Bianchi has combined his academic and clinical experience with parallel work in collaboration with pharmaceutical companies (Lepeft Windtree Therapeuticss in order to develop a targeted therapeutic approach to cardiovascular diseases through laboratory and clinical practice.

\section{Funding}

Dr Claudio Cavazza, Prassis Sigma Tau

- Windtree Therapeutics

\section{Collaborators}

- Citterio, Lorena - Genomics of Renal Diseases and Hypertension Unit, IRCCS San Raffaele Scientific Institute, Universita Vita Salute San Raffaele, Milano, Italy

- Cusi, Daniele - Institute of Biomedical Technologies Milano, National Research Bio4Dreams-B (CNR), Segrate, Milano, Italy; Bio4Dreams Scientific Unit,

- Ferrandi, Mara - Windtree Therapeutics, Warrington, PA, USA

- Lanzani, Chiara - Genomics of Renal Diseases and Hypertension Unit, IRCCS - Scioli Giuseppe Antonio - Hypertension and Cardiovascular Preventiano, Italy Center, Ospedale Ferdinando Veneziale, Isernia, Italy

- Glorioso, Nicola - Hypertension and Related Diseases Center, Department of Clinical and Experimental Medicine, University of Sassari, Sassari, Italy - Chiang, Chern-En - General Clinical Research Center, Taipei Veterans General Hospital and National Yang-Ming University, Taipei, Taiwan

Department of Internal Medicine, National Taiwan University Hospital and College of Medicine, National Taiwan University, Taipei, Taiwan - Lau, Lit-Fui - Zhaoke (Guangzhou) Ophthalmology Pharmaceutical Limited, Guangzhou, Chin

- Li, Xlaoyi - CVie Therapeutics, Taipei, Taiwan sità Vita Salute San Raffaele, Milano, Italy - Staessen, Jan A-Research Unit Hypertension and Cardiovascular Leuven, Leuven, Belgium

(

References

Citterio L, Bianchi G, Scioli GA, et al, (202 Antihypertensive treatment guided by
genetics: PEARL-HT, the randomized proo of-concept trial comparing rostafuroxin with losartan. The Pharmacogenomics Journal, $21(3), 346-358$
s41397-021-00214-y Manunta P, Ferrandi M, Cusi D, et al, (2016) Past and the Future Current Hypertension Report, 18, 24. doi.org/10.1007/s11906-0160632-y Ferrandi, M, Molinari, I, Torielli, L, et al, (201) Aductin- andi buabaintilated activity of rostafuroxin, part 1 . experimental studies. Science Translational Medicine, 2(59), 59 ra8 6 .
scitransimed. 3001815 Lanzani, C, Citterio, L, Glorioso, $N$, et al, variants predict the antihypertensive activity of rostafuroxin, part 2: clinical studies. Science Translational Medicine, 2(59), 59ra87. doil
org/101126/scitranslmed 3001814

\section{Personal Response}

What will Phase III trials for rostafuroxin

II Phase III trials should look for the types of bients caryostafuroxin in two profile 2 in comparison (to be discussed) with placebo (trial 1) and a standard treatment losartan or amlodipine (trial 2) type 1 - patients with so-called resistant
hypertension - that is, blood pressure above the normal range after one month of treatment when treated with three different types of drugs; and type 2 - newly to the first two separate treatments. Markers of cardiac and renal damage should also be measured. Composite primary endpoints include: number of therapy discontinuation, changes in his/ her quality of life, in office (or home) systolic blood pressure, the number of drugs and, 